\title{
THE VULKAN TECHNIQUE: A NOVEL OSTOMY-CLOSURE TECHNIQUE THAT REDUCES COMPLICATIONS AND OPERATIVE TIMES $\square$
}

\author{
Técnica Vulkan para fechamento de ileostomia e colostomia: efetiva redução de complicações e tempo operatório
}

Felix KRENZIEN ${ }^{1,2}$, Christian BENZING ${ }^{1}$, Fabian HARDERS ${ }^{3}$, Tido JUNGHANS ${ }^{3}$, Gyurdhan RASIM ${ }^{3}$, Claudia BOTHE ${ }^{3}$, Johann PRATSCHKE ${ }^{1}$, Ricardo ZORRON ${ }^{1,3}$

From the ${ }^{1}$ Center of Innovative Surgery (ZIC), Department of Surgery, Campus Virchow Klinikum and Campus Mitte, Charité-Universitätsmedizin Berlin, Berlin, Germany; ${ }^{2}$ Berlin Institut of Health $(\mathrm{BIH})$ Berlin, Germany; ${ }^{3}$ Department of General, Visceral, Thorax and Vascular Surgery, Clinic Bremerhaven Reinkenheide, Bremerhaven, Germany

HEADINGS - Vulkan technique. Ostomy. lleostomy. Colostomy, Surgical site infection.

\section{Correspondence::}

Ricardo Zorron

E-mail: ricardo.zorron@charite.de

Financial source:F.K. was supported by the Berlin Institute of Health $(\mathrm{BIH})$ and by the German Research Foundation (KR 4362/21).

Conflicts of interest: none

Received for publication: 16/11/2016 Accepted for publication: 11/04/2017

DESCRITORES - Técnica vulkan. Ostomia. lleostomia. Colostomia. Infecção do sítio cirúrgico.
ABSTRACT - Background: Ostomy reversals remain at high risk for surgical complications. Indeed, surgical-side infections due to bacterial contamination of the stoma lead to revision surgery and prolonged hospital stay. Aim: To describe the novel vulkan technique of ostomy reversal that aims to reduce operative times, surgical complications, and readmission rates. Methods: Ostomy closure was performed using the vulkan technique in all patients. This technique consists of external intestinal closure, circular skin incision and adhesiolysis, re-anastomosis, and closure of the subcutaneous tissue in three layers, while leaving a small secondary wound through which exudative fluid can be drained. The medical records of enterostomy patients were retrospectively reviewed from our hospital database. Results: The vulkan technique was successfully performed in 35 patients mainly by resident surgeons with $<5$ years of experience $(n=22 ; 62.8 \%)$. The ileostomy and colostomy closure times were 53 min (interquartile range [IQR], 41-68 min; $n=22$ ) and 136 min (IQR: 88-188 min; $n=13 ; p<0.001$ ), respectively. The median hospital stay was seven days (IQR: $5-14.5$ days); the length of hospital stay did not differ between ileostomy and colostomy groups. Major surgical complications occurred only in patients who underwent colostomy closure following the Hartmann procedure $(n=2)$; grade $\geq 1 \mathrm{ll} b$ according Clavien-Dindo classification. Conclusion: The vulkan technique was successfully applied in all patients with very low rates of surgical-site infections. Off note, residents with limited surgical experience mainly performed the procedure while operating time was less than one hour.

RESUMO - Racional: O procedimento de reversão de ileostomia ou colostomia após procedimento cirúrgico colônico permanecem com alto risco de complicações cirúrgicas. De fato, as infecções do sítio cirúrgico, devido à inerente contaminação bacteriana da operação, levam às operações de revisão e hospitalização prolongadas. Objetivo: O presente estudo visa descrever a técnica vulkan de reversão de ostomia, avaliando tempos operatórios, complicações cirúrgicas e taxas de readmissão. Métodos: 0 fechamento de ostomia foi realizado utilizando a técnica vulkan em todos os pacientes. Ela consiste em incisão cutânea circular, reanastomose, fechamento da aponeurose e fechamento do tecido subcutâneo em três camadas, deixando uma pequena ferida secundária através da qual se pode drenar o líquido exsudativo. A documentação dos pacientes com enterostomia foram revisadas retrospectivamente a partir da base de dados do hospital. Resultados: A técnica vulkan foi realizada com sucesso em 35 pacientes, principalmente por cirurgiões residentes com menos de cinco anos de experiência $(n=22$; $62,8 \%)$. Os tempos de ileostomia e fechamento da colostomia foram $53 \mathrm{~min}(41-68 \mathrm{~min} ; \mathrm{n}=22)$ e $136 \mathrm{~min}$ (88-188 min; $n=13 ; p<0,001)$, respectivamente. A média da permanência hospitalar foi de sete dias $(5-14,5$ dias); o tempo de internação não diferiu entre os grupos de ileostomia e colostomia. As complicações cirúrgicas maiores ocorreram somente nos pacientes que se submeteram ao fechamento da colostomia após o procedimento de Hartmann ( $n=2$, grau $\geq 11$ llb de acordo com a classificação de Clavien-Dindo). Conclusão: A técnica vulkan foi aplicada com sucesso em todos os pacientes com taxas muito baixas de infecções no local cirúrgico. Além disso, as operações foram realizadas principalmente por residentes com experiência cirúrgica limitada, resultando em tempos operatórios inferiores a uma hora.

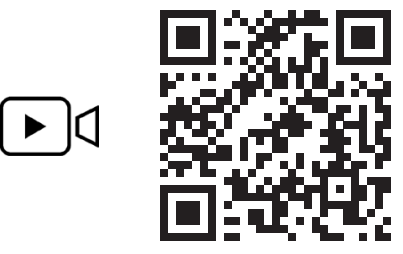

Supplementary video: https://youtu.be/yw-N-egaBNA

(cc) BY $\mathbf{B Y}$ This is an open-access article distributed under the terms of the Creative Commons Attribution License.

\section{INTRODUCTION}

$\mathrm{S}$ tomata are artificial openings on the body surface that lead to a hollow organ. In gastrointestinal surgery, stomata are created to divert the flow of feces away from a certain site and out of the body. Stomata can be reversed after patients have recovered and the initial pathology has resolved. The benefits of stomata for different underlying diseases have been widely reported ${ }^{14,17}$. In contrast, the surgical procedure of stomata closure is less well investigated, and can be affected by minor and major complications ${ }^{3}$. Although stoma reversal is widely performed in clinical practice, it is associated with high complication rates, which affect patient outcomes and increase hospitalization costs. The development of fistulas, fascial dehiscence, or small bowel obstruction can necessitate revision surgery and significantly impact the outcomes of patients with gastrointestinal stomata. Furthermore, surgical-site infections (SSIs) have been reported to occur in up to $41 \%$ of patients with stomata ${ }^{10,13}$.

To reduce stoma-related complications, various researchers have analyzed the different steps of the stomata reversal. Studies have been conducted to determine whether a hand-sewn anastomosis is superior to a stapled anastomosis. Based solely 
on randomized trials, stapledfunctional anastomoses are associated with fewer anastomotic leakages than hand-sewn anastomoses ${ }^{2}$. In addition, the conventional stoma-closure procedure has been performed laparoscopically. However, this is technically challenging, and is typically not performed by new or resident surgeons. Furthermore, owing to the complexity of the surgery, the procedure is prolonged, and has been associated with a mean operative time of up to $109 \mathrm{~min}^{8,11,12}$. Nevertheless, the laparoscopic procedure may be preferred in patients who require hernia repair and adhesiolysis, as it provides superior exposure of the surgical field and better access to the abdominal cavity.

Fascial and skin closure following stoma reversal are crucial for minimizing complications and facilitating recovery. Drainage tubes for the evacuation of exudative and suppurative fluids, and even delayed primary skin closure have been used to reduce the incidence of SSIs after stoma reversal ${ }^{8}$. Conventional closure through a linear incision enlarges the skin defect. In 1997, Banerjee et al. described the purse-string closure technique of stoma reversal ${ }^{1}$. Since then, systematic reviews have shown that this procedure does reduce SSI rates, but its impact on the length of hospital stay is questionable ${ }^{9,10,13}$. Interestingly, readmission rates have not been reported in most studies on stoma closure. One retrospective analysis of 351 enterostomy reversals reported a readmission rate of $12.5 \%$; moreover, this rate was linked to long operative times, and increased intraoperative complications and hospital stay.

To overcome the above shortcomings in ostomy closure, we developed technique for ostomy closure termed the "vulkan" technique. In this technique, intestinal re-anastomosis is followed by the circular closure of the subcutaneous tissue in layers with the retention of a small secondary defect through which exudative and suppurative fluids can be drained. This scenario is likened to a volcano or "vulkan" in German, hence the name. In this study, we validated the vulkan technique in a clinical series of 35 patients with loop ileostomy and end colostomy, in terms of patient outcomes, operative time, and surgical complications.

\section{METHODS}

\section{Study design}

This retrospective study was conducted at the Department of General, Visceral, Thoracic and Vascular Surgery, Bremerhaven Hospital, Germany. Between July 2011 and April 2013, 35 consecutive patients with protective loop ileostomy or end colostomy due to different underlying diseases underwent ostomy closure by the vulkan technique. The end colostomy had been created as part of the Hartmann procedure. All patients provided informed consent prior to undergoing the vulkan procedure.

The procedure was mainly performed by resident surgeons with less than five years of surgical experience. The medical records of the patients were documented in our hospital database. Follow-up data and readmission rates were assessed as well as operative times and surgical complications according to the Clavien-Dindo classification ${ }^{3}$. A single dose of an antibiotic (500 mg metronidazole/2 g cefuroxime) was prophylactically administered before the surgery.

\section{The vulkan technique of stoma closure}

Stoma closure was performed as follows: external closure of the intestinal mucosa to avoid fecal spillage (Figure 1A); circular incision around the ostomy to release adhesions and mobilize the bowel (Figure 1B); hand-sewn or stapled anastomosis (Figure 1C); and fascial closure with full-thickness running polydioxanone sutures (Figure 1D). Afterwards, sutures were placed in three consecutive layers, forming a triple crown. (Supplementary video, https://youtu. be/yw-N-egaBNA).

In contrast to the linear closure technique, drainage tubes were not placed after the vulkan procedure. Exudative and suppurative fluidswere evacuated through the small secondary defect remaining in the center of the wound, as all three circular sutures provide incomplete closure at each level.

\section{Statistical analysis}

Statistical analysis was performed using SPSS version 20 (IBM Corporation, Armonk, NY, USA). Continuous variables were expressed as medians, and categorical values were expressed as percentages. If applicable, the interquartile range (IQR; 25-75\%) was expressed. Differences between groups were analyzed using the Mann-Whitney $U$ test with Bonferroni correction or the Student t-test as appropriate. $P$ values smaller than 0.05 were considered significant.

\section{RESULTS}

The baseline characteristics of the patients are shown in Table 1. The vulkan technique was successfully performed in all 35 patients, of whom 13 (37.1\%) underwent colostomy reversal, and 22 (62.8\%) underwent ileostomy reversal. Most patients with ileostomy had colorectal cancer $(45.7 \%, n=16)$. All colostomies had been carried out in the course of a Hartmann procedure including a midline laparotomy followed by adhesiolysis. The vulkan procedure was performed mainly by resident surgeons $(62.8 \% ; n=22)$ with limited surgical experience (less than fiveyears).

TABLE 1 - Baseline characteristics of the study population

\begin{tabular}{|l|c|}
\hline Variable & Patients $(\mathbf{n}=35)$ \\
\hline Age (years) & $59.75(52-72)$ \\
\hline Gender & $19(54.3 \%)$ \\
\hline Male & $16(45.7 \%)$ \\
\hline Female & $7(5.2-12)$ \\
\hline Length of stay & $13(37.1 \%)$ \\
\hline Colostomy & $22(62.8 \%)$ \\
\hline lleostomy & $16(45.7 \%)$ \\
\hline Underlying disease & $6(17.1 \%)$ \\
\hline Colorectal cancer & $9(25.7$ \\
\hline Diverticulosis & \\
\hline Other &
\end{tabular}

The procedure time was significantly shorter for ileostomy closure than for colostomy closure $(p<0.001$; Figure $3 A)$. The mean procedure times for colostomy reversal and ileostomy reversal were $136 \mathrm{~min}$ (IQR: 88-188 $\mathrm{min}$ ) and $53 \mathrm{~min}$ (IQR: 41-68 min), respectively. Major surgical complications occurred only in the patients who underwent colostomy closure $(n=2$; grade $\geq$ IIlb; Figure 3B).

One patient developed fascial dehiscence caused by wound infection, and another suffered ischemic colitis. Both were treated using revision surgery. SSIs occurred in three patients (8.5\%; grade II), and were successfully managed with conventional dressings.

The cosmetic results were acceptable, and consisted of a small scar (Figure 4A) unlike the linear closure (Figure 4B). The wound shrunk in diameter, and the initial elevation of the tissue was decreased. The remaining wound opening was used to evacuate exudative fluids and thus prevent secondary infections. The mean length of stay following surgery was seven days (IQR: $5-14.5$ days), and did not significantly differ between the ileostomy ( 6.5 days; IQR: $5-13.3$ days) and colostomy groups (7.5 days; IQR: $5.5-15.5$ days). None of the patients was readmitted to the hospital due to complications of stoma reversal. 


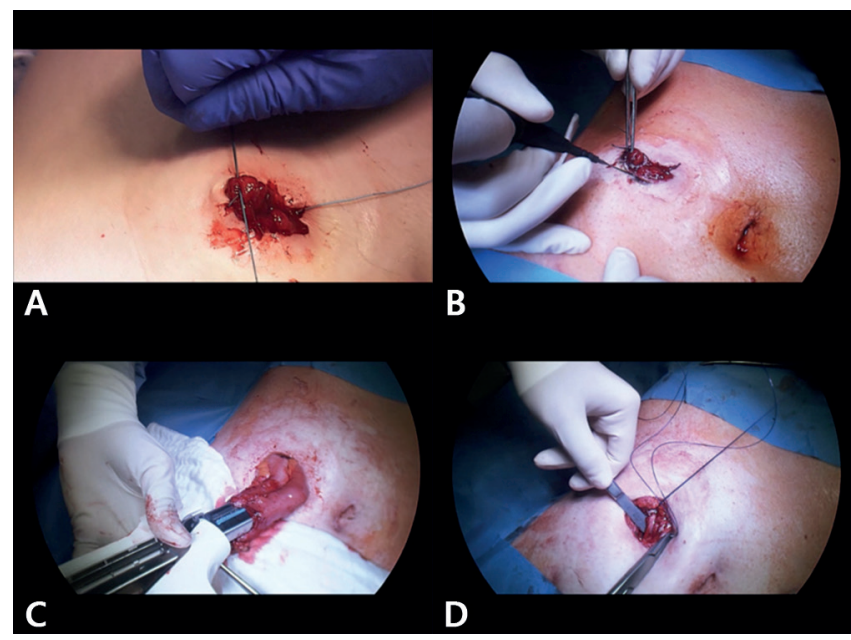

FIGURE 1 - The vulkan technique for ileostomy closure, part 1: A) a running suture (Vicryl 0-0) is temporarily placed at the mucosa level (without touching the skin) to close the ostomy and avoid stool spillage during the procedure; B) a circular incision is made around the ostomy to mobilize the ileum and it is important to make the incision at the border between the mucosa and the skin to avoid skin resection and create as small a wound defect as possible; C) progressive adhesiolysis of the bowel is performed followed by a stapled latero-lateral linear anastomosis; $\mathrm{D}$ ) the anastomosis can be oversewn with running polydioxanone (PDS) 4-0 sutures. Next, the abdominal fascia is closed with a full-thickness running suture (PDS 2-0).
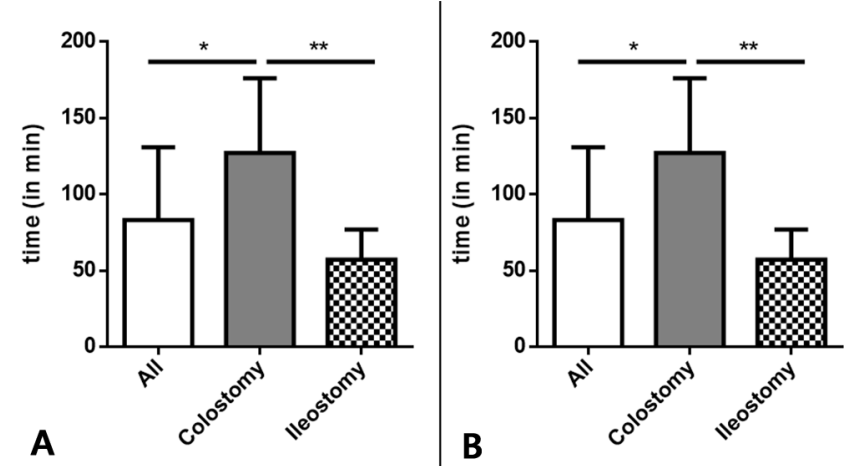

Values are expressed as mean $\pm \mathrm{SD} ;{ }^{*} \mathrm{p}<0.05 ;{ }^{*} \mathrm{p}<0.001$; Mann-Whitney U-test was used to compare groups

FIGURE 3 - Operative characteristics: A) operative times for ostomy reversal by the vulkan technique; B) postoperative morbidity as determined according to the ClavienDindo classification. Major complications were defined as complications with grade $\geq 1 \mathrm{ll}$.

DISCUSSION

The novel vulkan technique was successfully applied for enterostomy reversal in this clinical series of 35 patients. The technique was found to be simple and feasible as indicated by the proportion of resident surgeons who were able to successfully perform the surgery despite their limited surgical experience (less than five years). Moreover, the overall procedure time was less than $1 \mathrm{~h}$ in the case of ileostomy reversal. The cosmetic results were acceptable, and continuous evacuation of potentially exudative and suppurative fluids was enabled through the small wound defect.

The vulkan technique is characterized by the placement of consecutive, circular subcutaneous and subcuticular sutures following re-anastomosis and fascial closure. Indeed, there

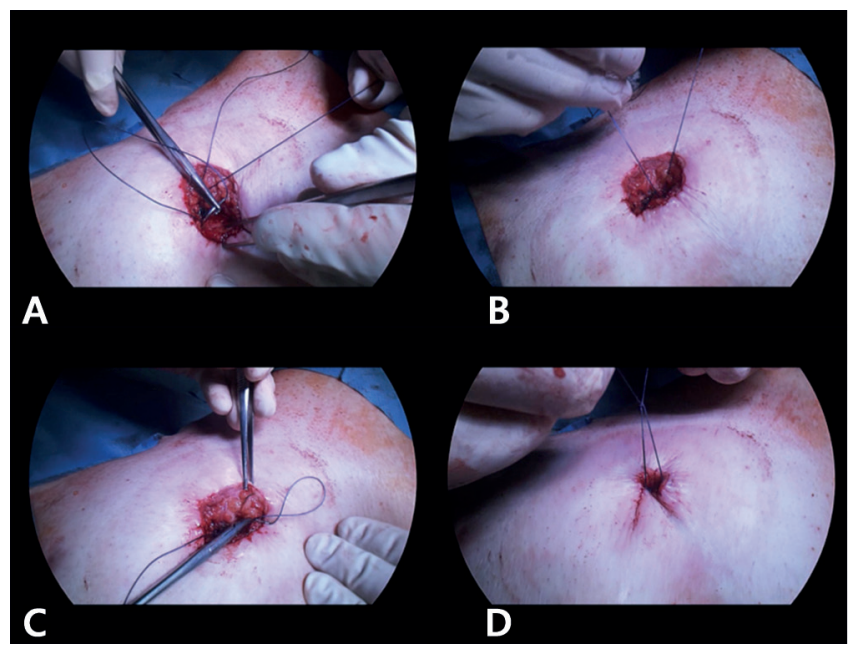

FIGURE 2 - The vulkan technique for ileostomy closure, part 2: $A$ and B) a circular pre-fascial subcutaneous suture is placed using Vicryl 1-0, and at this deep subcutaneous layer includes the fascial layer of the rectus abdominis to reduce the potential space for fluid accumulation; C) a second circular superficial subcutaneous suture is placed using Vicryl 1-0 as well; D) a third circular subcuticular suture is placed (Vicryl 1-0), forming a small wound opening. The three suture layers characterize the vulkan technique. Exudative fluid can be constantly evacuated from the remaining wound.

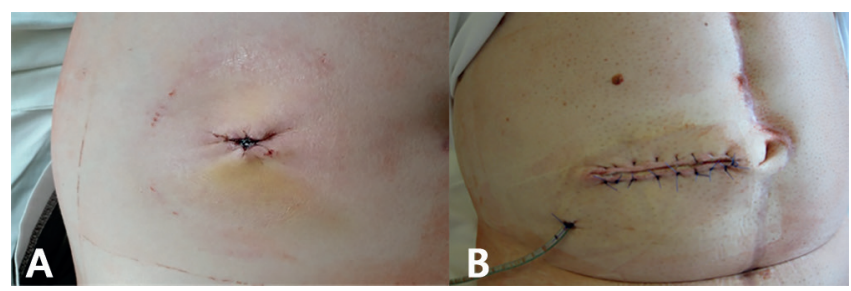

FIGURE 4 - Vulkan technique vs. conventional ostomy closure: A) the vulkan technique resulted in a small, circular scar, and exudative fluids were constantly drained through the remaining wound defect; B) cosmetic results of conventional enterostomy closure. Conventional linear closure leads to large scars, and exudative wound fluids can only be evacuated by the placement of a drainage tube.

are major differences between this technique and current stoma-closure techniques ${ }^{9}$. Conventional linear skin closure mostly involves a transverse linear incision. The skin is tightly adapted, and as fluid will remain enclosed underneath, the patient is prone to develop superinfection. Drainage can resolve the issue with fluid evacuation, but the incidence of SSIs after conventional linear closure remains high, up to $29.6 \%{ }^{10}$. In addition to the high complication rate, conventional linear closure is associated with a larger scar and inferior cosmetic results (Figure 4B) ${ }^{10}$. Purse-string stoma closure, like the vulkan technique, is performed using a circular incision around the enterostomy. However, the two techniques are distinct ${ }^{1}$. The vulkan technique involves subcutaneous sutures placed in three consecutive layers (triple crown). Moreover, the first (deepest) layer of sutures includes the fascial layer of the rectus abdominis to reduce the potential space for fluid accumulation. This is especially important in obese patients with a certain amount of subcutaneous fat. When single-layer closure is performed, large wound defects and SSIs will occur in high-risk patients. Interestingly, Banerjee et al. first proposed this open technique for ostomy closure but using only a single 
purse-string suture ${ }^{1}$. In systematic reviews, this procedure was able to reduce SSI rates as compared to linear closure,

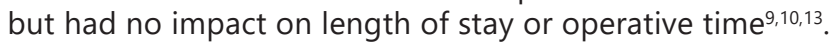

The vulkan procedure was newly introduced to our department. Thus, there was a learning curve for the procedure. Nevertheless, the operative time was less than $1 \mathrm{~h}$ for ileostomy reversal. The short operative time might reflect the ease of this technique, and this might be significant in an economical context. Prolonged operative times are known to be highly linked to readmission rates (odds ratio, 1.6) ${ }^{15}$ and increased complication rates ${ }^{15}$. In contrast, short operative times do not necessarily reflect superior clinical outcomes; for instance low recurrence rate has been reported for hernia repair when operative time was prolonged $(n=123,917)^{16}$. Taken together, the operative time for the vulkan technique solely indicates its feasibility and economic viability for ostomy closure in our specific clinical setting.

None of our patients required readmission after enterostomy closure. Although we cannot rule out the possibility that patients were admitted to other hospitals, most of the patients attended follow-up in our cancer program. Interestingly, readmission rates are rarely considered in clinical trials for stoma-closure techniques ${ }^{7}$. This bias profoundly affects the evaluation of closure techniques and the identification of all complications following the surgery. This issue seems to be a common problem in surgical and clinical trials. In contrast cost control and quality management studies necessitate the assessment of readmission rates ${ }^{4}$.

The anastomosis technique was not considered in our study and is not part of the vulkan technique. Whether a hand-sewn anastomosis is superior to stapled anastomosis remains uncertain. A Cochrane analysis of randomized trials reported that a stapledfunctional end-to-endileocolic anastomosis was associated with fewer complications than a hand-sewn anastomosis ${ }^{2}$. The anastomosis can also be created laparoscopically. The significance of laparoscopic reversal remains controversial, though selected patients may benefit from it ${ }^{18}$.

The present study has certain limitations. First, we did not compare our technique with conventional linear closure or PSC (Partial Subcutaneous Closure). This might be the most important drawback, since solely the vulkan technique was used for enterostomy reversal in this study. Clearly, it would be very interesting for upcoming clinical trials to validate this technique by comparing it with PSC or conventional closure. Second, this was a retrospective analysis, and was inferior to prospective studies. Third, the study was underpowered, as the number of patients was small, and all patients were treated by the proposed technique. Fourth, the vulkan technique was used for both ileostomy and colostomy closure. This led to differences in procedure times and complication rates. Compared to ileostomy closure, closure of the colostomy following the Hartmann procedure was associated with higher rates of major complications and significantly longer operative times. This difference is attributable to the necessity of a laparotomy and consecutive adhesiolysis in the colostomy patients, and is consistent with the current literature ${ }^{5}$.

\section{CONCLUSION}

Preliminary results of the novel vulkan technique for ostomy reversal indicate that this technique is feasible, safe, and easy to use, and yields good cosmetic results. These findings were underscored by the fact that most operations were performed by young surgeons, and yet, the overall operative time was less than $1 \mathrm{~h}$ for ileostomy closure. Further clinical trials are required to evaluate the proposed technique and compare it with established closure techniques.

\section{REFERENCES}

1. Banerjee A. Pursestring skin closure after stoma reversal. Diseases of the colon and rectum 1997; 40(8):993-4.

2. Choy PYG, Bissett IP, Docherty JG, Parry BR, Merrie AEH. Stapled versus handsewn methods for ileocolic anastomoses. The Cochrane database of systematic reviews 2007; (3):CD004320.

3. Clavien PA, Barkun J, Oliveira ML de, Vauthey JN, Dindo D, Schulick RD et al. The Clavien-Dindo classification of surgical complications: five-year experience. Annals of surgery 2009; 250(2):187-96.

4. FischerC, LingsmaHF, Marang-vandeMheen, PerlaJ, KringosDS, Klazinga NS, Steyerberg EW. Is the readmission rate a valid quality indicator? A review of the evidence. PloS one 2014; 9(11):e112282.

5. Geng HZ, Nasier D, Liu B, Gao H, Xu YK. Meta-analysis of elective surgical complications related to defunctioning loop ileostomy compared with loop colostomy after low anterior resection for rectal carcinoma. Annals of the Royal College of Surgeons of England 2015; 97(7):494-501.

6. Jackson TD, Wannares JJ, Lancaster RT, Rattner DW, Hutter MM. Does speed matter? The impact of operative time on outcome in laparoscopic surgery. Surgical endoscopy 2011; 25(7):2288-95.

7. Keller DS, Khorgami Z, Swendseid B, Khan S, Delaney CP. Identifying causesforhigh readmission rates afterstoma reversal.Surgical endoscopy 2014; 28(4):1263-8.

8. Lahat G, Tulchinsky H, Goldman G, Klauzner JM, Rabau M. Wound infection after ileostomy closure: a prospective randomized study comparing primary vs. delayed primary closure techniques. Techniques in coloproctology 2005; 9(3):206-8.

9. Li LT, Hicks SC, Davila JA, Kao LS, Berger RL, Arita NA et al. Circular closure is associated with the lowest rate of surgical site infection following stoma reversal: a systematic review and multiple treatment meta-analysis. Colorectal disease : the official journal of the Association of Coloproctology of Great Britain and Ireland 2014; 16(6):406-16.

10. McCartan DP, Burke JP, Walsh SR, Coffey JC. Purse-string approximation is superior to primary skin closure following stoma reversal: a systematic reviewandmeta-analysis. Techniquesincoloproctology2013;17(4):345-51.

11. Royds J, O'Riordan JM, MansourE, Eguare E, NearyP.Randomized clinical trial of the benefit of laparoscopy with closure of loop ileostomy. The British journal of surgery 2013; 100(10):1295-301.

12. RussekK,GeorgeJM,ZafarN, Cuevas-EstandiaP,FranklinM.Laparoscopic loop ileostomy reversal: reducing morbidity while improving functional outcomes. JSLS : Journal of the Society of Laparoendoscopic Surgeons / Society of Laparoendoscopic Surgeons 2011; 15(4):475-9.

13. Sajid MS, Bhatti MI, Miles WF. Systematic review and meta-analysis of published randomized controlled trials comparing purse-string vs conventional linear closure of the wound following ileostomy (stoma) closure. Gastroenterology report 2015; 3(2):156-61.

14. Tan WS, Tang CL, Shi L, Eu KW. Meta-analysis of defunctioning stomas in low anterior resection for rectal cancer. The British journal of surgery 2009; 96(5):462-72.

15. Tevis SE, Weber SM, Kent KC, Kennedy GD. Nomogram to Predict Postoperative Readmission in Patients Who Undergo General Surgery. JAMA surgery 2015; 150(6):505-10.

16. van der Linden W, Warg A, Nordin P. National register study of operating time and outcome in hernia repair. Archives of surgery (Chicago, III. : 1960) 2011; 146(10):1198-203.

17. WuS-W,MaC-C,Yang Y.Roleofprotectivestomain lowanterior resection for rectal cancer: a meta-analysis. World journal of gastroenterology 2014; 20(47):18031-7.

18. Young MT, Hwang GS, Menon G, Feldmann TF, Jafari MD, Jafari F et al. Laparoscopic Versus Open Loop Ileostomy Reversal: Is there an Advantage to a Minimally Invasive Approach? World journal of surgery 2015; 39(11):2805-11. 